\title{
The structure of fixed-point sets of uniformly lipschitzian semigroups
}

\author{
Jarosław Górnicki
}

Received: 30 August 2010 / Accepted: 22 February 2011 / Published online: 1 April 2011

(C) The Author(s) 2011. This article is published with open access at Springerlink.com

\begin{abstract}
In this paper, by asymptotic center techniques, we shown that the set of fixed points of a uniformly $k$-lipschitzian semigroup (one-parameter or left reversible semitopological) in a uniformly convex Banach space is a retract of the domain if $k$ is close to 1 . The results presented in this paper includes (among others, in the discrete situation) many known results as special cases.
\end{abstract}

Keywords One-parameter semigroup · Left reversible semigroup · Uniformly lipschitzian semigroup · Retraction - Asymptotic center · Fixed point - Uniformly convex Banach space

Mathematics Subject Classification (2000) Primary 47H10 - 47H20; Secondary $47 \mathrm{H} 09 \cdot 54 \mathrm{C} 15$

\section{Introduction}

We will consider a Banach spaces $E$ over the real field. Our notation and terminology are standard. Let $C$ be a nonempty bounded closed convex subset of $E$. We say that a mapping $T: C \rightarrow C$ is nonexpansive if

$$
\|T x-T y\| \leqslant\|x-y\| \text { for every } x, y \in C .
$$

The result of Bruck [2] asserts that if a nonexpansive mapping $T: C \rightarrow C$ has a fixed point in every nonempty closed convex subset of $C$ which is invariant under $T$ and if $C$ is convex and weakly compact, then $F(T)=\{x \in C: T x=x\}$, the set of fixed points, is a nonexpansive retract of $C$ (that is, there exists a nonexpansive mapping $R: C \rightarrow F(T)$ such that $R_{\mid F(T)}=I$ ). A few years ago, the Bruck result was extended by Domínguez Benavides

J. Górnicki ( $)$

Department of Mathematics, Rzeszów University of Technology,

P.O. Box 85, 35-959 Rzeszów, Poland

e-mail: gornicki@prz.edu.pl 
and Lorenzo Ramírez [4] to the case of asymptotically nonexpansive mappings if the space $E$ was sufficiently regular.

On the other hand it is known, the set of fixed points of $k$-lipschitzian mapping can be very irregular for any $k>1$.

Example 1 ([11]) Let $F$ be a nonempty closed subset of $C$. Fix $z \in F, 0<\varepsilon<1$ and put

$$
T x=x+\varepsilon \cdot \operatorname{dist}(x, F) \cdot(z-x), \quad x \in C .
$$

It is not difficult to see that $F(T)=F$ and the Lipschitz constant of $T$ tends to 1 if $\varepsilon \downarrow 0$.

In 1973, Goebel and Kirk [5] introduced the class of uniformly $k$-lipschitzian mappings and stated a relationship between the existence of fixed point for uniformly $k$-lipschitzian mappings and the Clarkson modulus of convexity $\delta_{E}$. Recall that a mapping $T: C \rightarrow C$ is uniformly $k$-lipschitzian, $k \geqslant 0$, if

$$
\left\|T^{n} x-T^{n} y\right\| \leqslant k\|x-y\| \text { for every } x, y \in C \text { and } n \in \mathbb{N} .
$$

Theorem 2 Let $E$ be a uniformly convex Banach space with modulus of convexity $\delta_{E}$ and let $C$ be a nonempty bounded closed convex subset of $E$. Suppose $T: C \rightarrow C$ is uniformly $k$-lipschitzian map and $k<\gamma$, where $\gamma>1$ is the unique solution of the equation

$$
\gamma\left(1-\delta_{E}\left(\frac{1}{\gamma}\right)\right)=1 \text {. }
$$

Then $F(T) \neq \varnothing$ (note that in a Hilbert space, $k<\gamma=\frac{1}{2} \sqrt{5}$, in $L^{p}$-spaces $(2 \leqslant p<$ $\left.\infty), k<\gamma=\left(1+2^{-p}\right)^{\frac{1}{p}}\right)$, and $F(T)$ is not only connected but even a retract of $C$ (see [11]).

In this paper we establish some results on the structure of fixed point sets for one-parameter uniformly $k$-lipschitzian semigroups and semi-topological uniformly $k$-lipschitzian semigroups in uniformly convex Banach spaces when $k$ is less than a constant bigger than the constant from Theorem 2.

\section{Uniformly convex Banach spaces}

Recall that the modulus of convexity $\delta_{E}$ is the function $\delta_{E}:[0,2] \rightarrow[0,1]$ defined by

$$
\delta_{E}(\varepsilon)=\inf \left\{1-\frac{1}{2}\|x+y\|:\|x\| \leqslant 1,\|y\| \leqslant 1,\|x-y\| \geqslant \varepsilon\right\}
$$

and that the space $E$ is uniformly convex if $\delta_{E}(\varepsilon)>0$ for $\varepsilon>0$. A Hilbert space $H$ is uniformly convex. This fact is a direct consequence of parallelogram identity. It is well known that $\delta_{E}$ is continuous on $[0,2)$ and strictly increasing in uniformly convex Banach spaces [6, Lemma 5.1].

Recall the concept and the notion of asymptotic center due to Edelstein, see [1,6]. Let $C$ be a nonempty closed convex subset of a Banach space $E$, and $G$ an unbounded subset of $[0,+\infty)$ such that $t+h \in G$ for all $t, h \in G$ and $t-h \in G$ for all $t, h \in G$ with $t \geqslant h$ (i.e., $G=[0,+\infty), G=[0,+\infty) \cap \mathbb{Q}$ or $G=\mathbb{N}_{0}$, the set of nonnegative integers), and let $\left\{x_{t}: t \in G\right\}$ be a bounded family of elements of $E$. Then the asymptotic radius and asymptotic center of $\left\{x_{t}\right\}_{t \in G}$ with respect to $C$ are the number

$$
r\left(C,\left\{x_{t}\right\}\right):=\inf _{y \in C}\left(\limsup _{G \ni t \rightarrow \infty}\left\|y-x_{t}\right\|\right)
$$


and the (possibly empty) set

$$
A\left(C,\left\{x_{t}\right\}\right):=\left\{y \in C: \limsup _{G \ni t \rightarrow \infty}\left\|y-x_{t}\right\|=r\left(C,\left\{x_{t}\right\}\right)\right\},
$$

respectively. It is well known that if $E$ is reflexive, then $A\left(C,\left\{x_{t}\right\}\right)$ is bounded closed convex and nonempty, and if $E$ is uniformly convex, then $A\left(C,\left\{x_{t}\right\}\right)$ consist only a single point, $\{z\}=A\left(C,\left\{x_{t}\right\}\right)$, i.e., other words $z \in C$ is the unique point which minimizes functional

$$
\limsup _{G \ni t \rightarrow \infty}\left\|y-x_{t}\right\|
$$

over $y$ in $C$.

Suppose $\mathcal{F}=\left\{T_{s}: s \in G\right\}$ is a one-parameter uniformly k-lipschitzian semigroup on $C$, i.e., a family of self-mappings on $C$ satisfying the conditions:

1. $T_{s+h} x=T_{s} T_{h} x$ for all $s, h \in G$ and $x \in C$,

2. for each $x \in C$, the mapping $s \rightarrow T_{s} x$ from $G$ into $C$ is continuous when $G$ has the relative topology of $[0,+\infty)$,

3. for each $s \in G,\left\|T_{s} x-T_{s} y\right\| \leqslant k\|x-y\|$ for all $x, y \in C$.

Let $A: C \rightarrow C$ denote a mapping which associates with a given $x \in C$ a unique $z \in$ $A\left(C,\left\{T_{t} x\right\}\right)$, that is, $z=A x$. Now we generalize to uniformly $k$-lipschitzian semigroups the lemma due to Sędłak and Wiśnicki [11]. This lemma is crucial to our results.

Lemma 3 Let $E$ be a uniformly convex Banach space and let $C$ be a nonempty bounded closed convex subset of $E$. Then the mapping $A: C \rightarrow C$ is continuous.

Proof On the contrary, suppose that there exists $x_{0} \in C$ and $\varepsilon_{0}>0$ such that:

for all $\eta>0$ there exists $x_{1} \in C$ such that $\left\|x_{1}-x_{0}\right\|<\eta$ and $\left\|z_{1}-z_{0}\right\| \geqslant \varepsilon_{0}$, where $\left\{z_{0}\right\}=A\left(C,\left\{T_{t} x_{0}\right\}\right),\left\{z_{1}\right\}=A\left(C,\left\{T_{t} x_{1}\right\}\right)$.

Fix $\eta>0$ and take $x_{1} \in C$ such that

$$
\left\|x_{1}-x_{0}\right\|<\eta \text { and }\left\|z_{1}-z_{0}\right\| \geqslant \varepsilon_{0} .
$$

Let

$$
\begin{aligned}
& R_{0}=r\left(C,\left\{T_{t} x_{0}\right\}\right)=\inf _{y \in C}\left(\limsup _{G \ni t \rightarrow+\infty}\left\|y-T_{t} x_{0}\right\|\right), \\
& R_{1}=r\left(C,\left\{T_{t} x_{1}\right\}\right)=\inf _{y \in C}\left(\limsup _{G \ni t \rightarrow+\infty}\left\|y-T_{t} x_{1}\right\|\right)
\end{aligned}
$$

and

$$
R=\limsup _{G \ni t \rightarrow+\infty}\left\|z_{1}-T_{t} x_{0}\right\|
$$

Notice that

$$
R_{0}<R \text {. }
$$

Choose $\varepsilon>0$. Then exists $s(\varepsilon) \in G$ that

$$
\left\{\begin{array}{l}
\left\|z_{1}-T_{t} x_{0}\right\|<R+\varepsilon \\
\left\|z_{0}-T_{t} x_{0}\right\|<R_{0}+\varepsilon<R+\varepsilon \\
\left\|z_{0}-z_{1}\right\| \geqslant \varepsilon_{0}
\end{array}\right.
$$

for all $t \in G$ and $t \geqslant s(\varepsilon)$. 
It follows by (2) and the properties of $\delta_{E}$ that for $G \ni t \geqslant s(\varepsilon)$,

$$
\left\|T_{t} x_{0}-\frac{z_{1}+z_{0}}{2}\right\| \leqslant\left(1-\delta_{E}\left(\frac{\varepsilon_{0}}{R+\varepsilon}\right)\right)(R+\varepsilon)
$$

and hence

$$
R_{0}<\limsup _{G \ni t \rightarrow+\infty}\left\|T_{t} x_{0}-\frac{z_{1}+z_{0}}{2}\right\| \leqslant\left(1-\delta_{E}\left(\frac{\varepsilon_{0}}{R+\varepsilon}\right)\right)(R+\varepsilon) .
$$

Moreover for $t \in G$, from triangle inequality we have

$$
\left\|T_{t} x_{0}-z_{1}\right\| \leqslant\left\|T_{t} x_{o}-T_{t} x_{1}\right\|+\left\|T_{t} x_{1}-z_{1}\right\| \leqslant k\left\|x_{0}-x_{1}\right\|+R_{1}+\varepsilon,
$$

and hence

$$
R=\limsup _{G \ni t \rightarrow+\infty}\left\|T_{t} x_{0}-z_{1}\right\| \leqslant k \eta+R_{1}+\varepsilon
$$

Similarly,

$$
R_{1}<\limsup _{G \ni t \rightarrow+\infty}\left\|T_{t} x_{1}-z_{0}\right\| \leqslant k \eta+R_{1}+\varepsilon .
$$

From (4) and (5), we have

$$
R \leqslant k \eta+R_{1}+\varepsilon<2 k \eta+2 \varepsilon+R .
$$

Combining (6) with (3) and applying the monotonicity of $\delta_{E}$, we obtain

$$
R_{0}<\left(1-\delta_{E}\left(\frac{\varepsilon_{0}}{2 k \eta+3 \varepsilon+R_{0}}\right)\right)\left(2 k \eta+3 \varepsilon+R_{0}\right) .
$$

Letting $\eta, \varepsilon \downarrow 0$, and using the continuity of $\delta_{E}$, we conclude that

$$
1 \leqslant\left(1-\delta_{E}\left(\frac{\varepsilon_{0}}{R_{0}}\right)\right)<1 .
$$

This contradiction proves the continuity of the mapping $A$.

This result can be extend to left reversible semigroups. Now let $J$ be a semi-topological semigroup, i.e., $J$ is a semigroup with a Hausdorff topology such that for each $a \in J$ the mapping $s \rightarrow a \cdot s$ and $s \rightarrow s \cdot a$ from $J$ to $J$ are continuous. A semi-topological semigroup $J$ is said to be left reversible if any two closed right ideals have non-void intersection. (This latter is automatically fulfilled if, for example $J$ is commutative, and in particulary if $J=[0,+\infty)$.) In this case $(J, \leqslant)$ is a directed system when the binary relation " $\leqslant$ " on $J$ is defined by $a \leqslant b$ if and only if $\{a\} \cup \overline{a J} \supseteq\{b\} \cup \overline{b J}$.

Let $\left\{x_{a}: a \in J\right\}$ be a bounded net in uniformly convex Banach space $E$ and let $C$ a nonempty closed convex subset of $E$. For a fixed $p>1$, let us set

$$
r(x)=\inf _{b \in J} \sup _{a \geqslant b}\left\|x_{a}-x\right\|^{p} \text { and } r=\inf _{x \in C} r(x) .
$$

Then we have a unique point $z \in C$ (called the asymptotic center of the net $\left\{x_{a}\right\}$ in $C$ ) such that $r(z)=r$.

Let $C$ be a nonempty bounded closed convex subset of a Banach space $E$, let $J$ be a left reversible semi-topological semigroup, and let $\mathcal{T}=\left\{T_{s}: s \in J\right\}$ be a family of selfmappings of $C$ into itself. Then $\mathcal{T}$ is said to be a left reversible semi-topological uniformly $k$-lipschitzian semigroup on $C$ if the following conditions are satisfied: 
$1^{\prime} . T_{t s} x=T_{t} T_{s} x$ for all $t, s \in J$ and $x \in C$,

$2^{\prime}$. the mapping $(s, x) \rightarrow T_{s} x$ from $J \times C$ into $C$ is continuous when $J \times C$ has the product topology,

$3^{\prime}$. for each $s \in J,\left\|T_{s} x-T_{s} y\right\| \leqslant k\|x-y\|$ for all $x, y \in C$.

Remark 4 For such a family of mappings Lemma 3 remains true.

Normal structure plays essential role in some problems of metric fixed point theory. Let $C$ be a nonempty bounded set in a Banach space $E$. We put

$$
r(C)=\inf _{x \in C}\left(\sup _{y \in C}\|x-y\|\right) .
$$

This number is called the Chebyshev radius of $A$.

A Banach space $E$ is said to have uniformly normal structure (UNS) if for some $c \in(0,1)$ and every bounded closed convex subset $C \subset E$ with diam $C>0$, it has

$$
r(C) \leqslant c \cdot \operatorname{diam} C .
$$

The normal structure coefficient (also called the Jung constant) was defined by Bynum [3] in the following way

$$
N(E):=\inf \left\{\frac{\operatorname{diam} C}{r(C)}\right\}
$$

where the infimum is taken over all bounded closed convex sets $C \subset E$ with diam $C>0$. Clearly, the condition $N(E)>1$ characterizes spaces $E$ with UNS. It is well known that all uniformly convex Banach spaces possess UNS [10, Theorem 5.12]. It is difficult to calculate the normal structure coefficient in an arbitrary Banach space. However, for a Hilbert space, $N(H)=\sqrt{2}$, and $N\left(l^{p}\right)=N\left(L^{p}\right)=\min \left\{2^{\frac{1}{p}}, 2^{1-\frac{1}{p}}\right\}$ for $1<p<\infty$, see $[1,10]$.

The following lemma can be proved in exactly the same way as Lim [8, Theorem 1] for sequences and the proof is thus omitted here.

Lemma 5 Let $E$ be a Banach space with UNS. Then for every bounded family $\left\{x_{t}\right\}_{t \in G}$ of elements of $E$ there exists $y$ in $\overline{\operatorname{conv}}\left\{x_{t}: t \in G\right\}$ such that

$$
\limsup _{G \ni t \rightarrow+\infty}\left\|y-x_{t}\right\| \leqslant \frac{1}{N(E)} \cdot \lim _{G \ni t \rightarrow+\infty}\left(\sup \left\{\left\|x_{i}-x_{j}\right\|: t \leqslant i, j \in G\right\}\right) .
$$

Now we improve the fixed point theorem due to Tan and $\mathrm{Xu}$ [12, Theorem 3.5].

Theorem 6 Let $E$ be a uniformly convex Banach space and let $C$ be a nonempty bounded closed convex subset of E. Suppose $\mathcal{F}=\left\{T_{s}: s \in G\right\}$ is a one-parameter uniformly $k$-lipschitzian semigroup on $C$ with $k<\alpha$, where $\alpha>1$ is the unique solution of the equation

$$
\alpha^{2} \cdot \delta_{E}^{-1}\left(1-\frac{1}{\alpha}\right) \cdot \frac{1}{N(E)}=1
$$

(in a Hilbert space $\left.\alpha=(\sqrt{3}-1)^{-\frac{1}{2}}>\frac{1}{2} \sqrt{5}\right)$. Then

$$
F(\mathcal{F})=\left\{x \in C: T_{s} x=x \text { for all } s \in G\right\} \neq \varnothing
$$

and $F(\mathcal{F})$ is a retract of $C$. 
Suppose $E$ is uniformly convex Banach space and $\alpha>1$ is the unique solution of Eq. (7). Then $\gamma<\alpha$, where $\gamma>1$ is the unique solution of Eq. (1), see [12, Lemma 3.3].

Proof The proof of existence $z$ in $C$ such that $T_{s} z=z$ for all $s \in G$, based on the Lemma 5, is given in [12, Theorem 3.5] or in [7, Theorem 4]. In this proof by induction we define a sequence $\left\{x_{n}\right\}_{n=0,1,2, \ldots}$ in $C$ in the following manner

$$
x_{0}=x \quad \text { and } \quad x_{n+1}=A\left(C,\left\{T_{t} x_{n}\right\}\right)=A^{n+1} x, \quad n=0,1, \ldots
$$

$\left(z=\lim _{n \rightarrow+\infty} x_{n}\right)$. Thus by the inequalities

$$
\begin{gathered}
d\left(x_{n}\right)=\sup _{t \in G}\left\|T_{t} x_{n}-x_{n}\right\| \leqslant d\left(x_{n-1}\right) \leqslant B^{n} d(x), \\
\left\|x_{n+1}-x_{n}\right\| \leqslant\left(\frac{k}{N(E)}+1\right) B^{n} d(x) \rightarrow 0 \quad \text { as } n \rightarrow+\infty,
\end{gathered}
$$

where $B=\frac{k^{2}}{N(E)} \delta_{E}^{-1}\left(1-\frac{1}{k}\right)<1$, we have

$$
\left\|A^{n+1} x-A^{n} x\right\| \leqslant\left(\frac{k}{N(E)}+1\right) B^{n} d(x) \leqslant\left(\frac{k}{N(E)}+1\right) B^{n} \operatorname{diam} C
$$

for $n=1,2, \ldots$ So

$$
\sup _{x \in C}\left\|A^{i} x-A^{m} x\right\| \leqslant\left(\frac{k}{N(E)}+1\right) \frac{B^{m}}{1-B} \operatorname{diam} C \rightarrow 0 \text { if } i, m \rightarrow+\infty,
$$

which implies that the sequence $\left\{A^{m} x\right\}_{m=1,2, \ldots}$ converges uniformly to a function

$$
R x=\lim _{m \rightarrow \infty} A^{m} x, \quad x \in C .
$$

It follows from Lemma 3, that $R: C \rightarrow C$ is continuous. Moreover,

$$
\begin{aligned}
& \left\|R x-T_{S} R x\right\| \\
& \quad \leqslant\left\|R x-A^{m} x\right\|+\left\|A^{m} x-T_{s+h} A^{m} x\right\|+\left\|T_{s+h} A^{m} x-T_{s} A^{m} x\right\|+\left\|T_{s} A^{m} x-T_{S} R x\right\| \\
& \leqslant(1+k)\left\|R x-A^{m} x\right\|+\left\|A^{m} x-T_{s+h} A^{m} x\right\|+k\left\|T_{h} A^{m} x-A^{m} x\right\| \\
& \leqslant(1+k)\left\|R x-A^{m} x\right\|+(1+k) \cdot d\left(A^{m} x\right) \\
& \quad \leqslant(1+k)\left\|R x-A^{m} x\right\|+(1+k) \cdot B^{m} \cdot \operatorname{diam} C \rightarrow 0 \text { as } m \rightarrow+\infty
\end{aligned}
$$

and $R x=T_{s} R x$ for all $s \in G$ and $x \in C$. Thus $R$ is a retraction of $C$ onto $F(\mathcal{F})$.

This result can be sharpened in some uniformly convex Banach spaces, for example in a Hilbert space and in $L^{p}$-spaces $(1<p<\infty)$.

\section{3 p-Uniformly convex Banach spaces}

Let $p>1$ be a real number. A Banach space $E$ is said to be $p$-uniformly convex (or $E$ is said to have the modulus of convexity of power type $p$ ) if there exists a constant $d>0$ such that the modulus of convexity $\delta_{E}(\varepsilon) \geqslant d \cdot \varepsilon^{p}$ for $0 \leqslant \varepsilon \leqslant 2$. We note that a Hilbert space is 2-uniformly convex (indeed, $\delta_{H}(\varepsilon)=1-\sqrt{1-\left(\frac{\varepsilon}{2}\right)^{2}} \geqslant \frac{1}{8} \varepsilon^{2}$ ) and an $L^{p}$-space $(1<p<\infty)$ is $\max \{p, 2\}$-uniformly convex.

In $[9,14]$ the following result was proved. 
Theorem 7 Let $p>1$ be a real number and let $E$ be a p-uniformly convex Banach space. Then there exists a constant $c_{p}>0$ such that

$$
\|\lambda x+(1-\lambda) y\|^{p} \leqslant \lambda\|x\|^{p}+(1-\lambda)\|y\|^{p}-c_{p} \cdot W_{p}(\lambda) \cdot\|x-y\|^{p}
$$

for all $x, y \in E, 0 \leqslant \lambda \leqslant 1$, where $W_{p}(\lambda)=\lambda(1-\lambda)^{p}+\lambda^{p}(1-\lambda)$.

Let $H$ be a Hilbert space, then

$$
\|\lambda x+(1-\lambda) y\|^{2}=\lambda\|x\|^{2}+(1-\lambda)\|y\|^{2}-\lambda(1-\lambda)\|x-y\|^{2}
$$

for all $x, y \in H, 0 \leqslant \lambda \leqslant 1$.

When $E$ is an $L^{p}$-space, we have the following

Theorem 8 Suppose $E$ is an $L^{p}$-space.

(a) If $1<p \leqslant 2$, then

$$
\|\lambda x+(1-\lambda) y\|^{2} \leqslant \lambda\|x\|^{2}+(1-\lambda)\|y\|^{2}-(p-1) \cdot \lambda \cdot(1-\lambda) \cdot\|x-y\|^{2}
$$

for all $x, y \in E$ and $0 \leqslant \lambda \leqslant 1\left(c_{p}=p-1\right)$;

(b) If $2<p<\infty$, then

$$
\|\lambda x+(1-\lambda) y\|^{p} \leqslant \lambda\|x\|^{p}+(1-\lambda)\|y\|^{p}-c_{p} \cdot W_{p}(\lambda) \cdot\|x-y\|^{p}
$$

for all $x, y \in E, 0 \leqslant \lambda \leqslant 1$, where $W_{p}(\lambda)=\lambda(1-\lambda)^{p}+\lambda^{p}(1-\lambda)$ and

$$
c_{p}=\frac{1+t_{p}^{p-1}}{\left(1+t_{p}\right)^{p-1}}=(p-1)\left(1+t_{p}\right)^{2-p}
$$

with $t_{p}$ being the unique solution of the equation

$$
(p-2) t^{p-1}+(p-1) t^{p-2}-1=0, \quad 0<t<1 .
$$

All constant appeared in the above inequalities are the best possible.

In the following theorem we improve the fixed point theorem due to Xu [14] from point of view of the structure of the set of fixed points.

Theorem 9 Let $p>1$ be a real number and let $E$ be a p-uniformly convex Banach space, $C$ a nonempty bounded closed convex subset of $E$. Suppose $\mathcal{F}=\left\{T_{s}: s \in G\right\}$ is a oneparameter uniformly $k$-lipschitzian semigroup on $C$ with $k<k_{p}$, where $k_{p}>1$ is the unique solution of the equation

$$
\left(t^{p}\right)^{2}-t^{p}-[N(E)]^{p} \cdot c_{p}=0, \quad t \in(0,+\infty),
$$

i.e., $k_{p}=\left[\frac{1}{2}\left(1+\sqrt{1+4 \cdot c_{p} \cdot[N(E)]^{p}}\right)\right]^{\frac{1}{p}}$. Then $F(\mathcal{F}) \neq \varnothing$ and $F(\mathcal{F})$ is a retract of $C$.

Proof We may assume that $k \geqslant 1$ since if $k<1$, the well known Banach Contraction Principle guarantees a fixed point of $\mathcal{F}$.

For an $x=x_{0} \in C$, we can inductively define a sequence $\left\{x_{m}\right\}_{m=1,2, \ldots}$ in $C$ in the following way: $x_{m+1}$ is the asymptotic center of the sequence $\left\{T_{t} x_{m}\right\}_{t \in G}$, that is, $x_{m+1}$ is the unique point in $C$ that minimizes the functional

$$
\limsup _{G \ni t \rightarrow+\infty}\left\|y-T_{t} x_{m}\right\|
$$


over $y$ in $C$. For each $m \geqslant 0$, we set

$$
r_{m}=\limsup _{G \ni t \rightarrow+\infty}\left\|x_{m+1}-T_{t} x_{m}\right\| \text { and } d\left(x_{m}\right)=\sup _{t \in G}\left\|x_{m}-T_{t} x_{m}\right\| .
$$

Then by Lemma 5, we have

$$
\begin{aligned}
r_{m} & \leqslant \frac{1}{N(E)} \cdot \lim _{G \ni t \rightarrow+\infty}\left(\sup \left\{\left\|T_{s+h} x_{m}-T_{s} x_{m}\right\|: t \leqslant s \in G \text { and } h \in G\right\}\right) \\
& \leqslant \frac{k}{N(E)} \cdot \sup _{t \in G}\left\|T_{t} x_{m}-x_{m}\right\|=\frac{k}{N(E)} \cdot d\left(x_{m}\right)
\end{aligned}
$$

for $m=0,1,2, \ldots$

Now from Theorem 7 for each fixed $m \geqslant 0$ and $s, h \in G$, we have

$$
\begin{aligned}
& \left\|\lambda x_{m+1}+(1-\lambda) T_{s} x_{m+1}-T_{s+h} x_{m}\right\|^{p}+c_{p} \cdot W_{p}(\lambda) \cdot\left\|x_{m+1}-T_{s} x_{m+1}\right\|^{p} \\
& \quad \leqslant \lambda\left\|x_{m+1}-T_{s+h} x_{m}\right\|^{p}+(1-\lambda)\left\|T_{s+h} x_{m}-T_{s} x_{m+1}\right\|^{p} \\
& \quad \leqslant \lambda\left\|x_{m+1}-T_{s+h} x_{m}\right\|^{p}+(1-\lambda) \cdot k^{p} \cdot\left\|T_{h} x_{m}-x_{m+1}\right\|^{p} .
\end{aligned}
$$

Taking the limit superior as $G \in h \rightarrow+\infty$ and nothing that $x_{m+1}$ is the asymptotic center of the sequence $\left\{T_{t} x_{m}\right\}_{t \in G}$, we obtain for each $s \in G$,

$$
r_{m}^{p}+c_{p} \cdot W_{p}(\lambda) \cdot\left\|x_{m+1}-T_{s} x_{m+1}\right\|^{p} \leqslant\left[\lambda+(1-\lambda) \cdot k^{p}\right] \cdot r_{m}^{p},
$$

and

$$
\left\|x_{m+1}-T_{s} x_{m+1}\right\|^{p} \leqslant \frac{(1-\lambda)\left(k^{p}-1\right)}{c_{p} \cdot W_{p}(\lambda)} \cdot r_{m}^{p} .
$$

It then follows that

$$
\left[d\left(x_{m+1}\right)\right]^{p} \leqslant \frac{(1-\lambda)\left(k^{p}-1\right)}{c_{p} \cdot W_{p}(\lambda)} \cdot r_{m}^{p} \leqslant \frac{(1-\lambda)\left(k^{p}-1\right)}{c_{p} \cdot W_{p}(\lambda)} \cdot \frac{k^{p}}{[N(E)]^{p}} \cdot\left[d\left(x_{m}\right)\right]^{p} .
$$

Letting $\lambda \uparrow 1$, we get

$$
\left[d\left(x_{m+1}\right)\right]^{p} \leqslant \frac{k^{p}\left(k^{p}-1\right)}{c_{p} \cdot[N(E)]^{p}} \cdot\left[d\left(x_{m}\right)\right]^{p}
$$

and

$$
d\left(x_{m+1}\right) \leqslant\left(\frac{k^{p}\left(k^{p}-1\right)}{c_{p} \cdot[N(E)]^{p}}\right)^{\frac{1}{p}} \cdot d\left(x_{m}\right)=B_{p} \cdot d\left(x_{m}\right), \quad m=0,1,2, \ldots,
$$

where $B_{p}=\left(\frac{k^{p}\left(k^{p}-1\right)}{c_{p} \cdot[N(E)]^{p}}\right)^{\frac{1}{p}}<1$ by assumption of the theorem. In a similar way, we obtain

$$
d\left(x_{m+1}\right) \leqslant B_{p} \cdot d\left(x_{m}\right) \leqslant \cdots \leqslant\left(B_{p}\right)^{m+1} \cdot d(x) .
$$

Since

$$
\left\|x_{m+1}-x_{m}\right\| \leqslant\left\|x_{m+1}-T_{t} x_{m}\right\|+\left\|T_{t} x_{m}-x_{m}\right\|,
$$

so taking the limit superior as $G \ni t \rightarrow+\infty$, we get by (8), (9),

$$
\begin{aligned}
\left\|x_{m+1}-x_{m}\right\| \leqslant r_{m}+d\left(x_{m}\right) & \leqslant\left(\frac{k}{N(E)}+1\right) d\left(x_{m}\right) \\
& \leqslant\left(\frac{k}{N(E)}+1\right) \cdot\left(B_{p}\right)^{m+1} \cdot d(x) \rightarrow 0 \text { as } m \rightarrow+\infty,
\end{aligned}
$$


and we see that $\left\{x_{m}\right\}$ is norm Cauchy and hence strong convergent. Let $z=\lim _{m \rightarrow \infty} x_{m}$. Then we have

$$
\begin{aligned}
\left\|T_{s} z-z\right\| & \leqslant\left\|T_{s} z-T_{s} x_{m}\right\|+\left\|T_{s} x_{m}-x_{m}\right\|+\left\|x_{m}-z\right\| \\
& \leqslant(1+k)\left\|x_{m}-z\right\|+d\left(x_{m}\right) \\
& \leqslant(1+k)\left\|x_{m}-z\right\|+\left(B_{p}\right)^{m} \cdot d(x) \rightarrow 0 \text { as } m \rightarrow+\infty,
\end{aligned}
$$

and $T_{s} z=z$ for all $s \in G$.

The proof of the retraction $R: C \rightarrow F(\mathcal{F})$ can be proved in exactly the same way as in the proof of Theorem 6 .

Corollary 10 Let $H$ be a Hilbert space, $C$ a nonempty bounded closed convex subset of $H$ and $\mathcal{F}=\left\{T_{s}: s \in G\right\}$ be a one-parameter uniformly $k$-lipschitzian semigroup on $C$ with $k<\sqrt{2}$. Then $F(\mathcal{F}) \neq \varnothing$ and $F(\mathcal{F})$ is a retract of $C$.

Corollary 11 Let C be a nonempty bounded closed convex subset of $L^{p}$-space $(1<p<\infty)$ and $\mathcal{F}=\left\{T_{s}: s \in G\right\}$ be a one-parameter uniformly $k$-lipschitzian semigroup on $C$. Suppose $k<\sqrt{\frac{1}{2}+\frac{1}{2} \sqrt{1+(p-1) 4^{2-\frac{1}{p}}}}$ if $1<p \leqslant 2$ (in particular, in $L^{2}$-space, $k<\sqrt{2}$ ) and $k<\left(\frac{1}{2}+\frac{1}{2} \sqrt{1+8 \cdot c_{p}}\right)^{\frac{1}{p}}$ if $2<p<+\infty$ (here $c_{p}$ is as in Theorem $\left.8(b)\right)$. Then $F(\mathcal{F}) \neq \varnothing$ and $F(\mathcal{F})$ is a retract of $C$.

For left reversible semi-topological semigroup we have the following [13, Lemma 3].

Lemma 12 Let $E$ be p-uniformly convex Banach space for some $p>1, C$ a nonempty bounded closed convex subset of $E$. Let $J$ be a left reversible semi-topological semigroup and $\left\{x_{a}: a \in J\right\}$ be a net in $C$. Let us set

$$
r(x)=\inf _{b \in J} \sup _{a \geqslant b}\left\|x_{a}-x\right\|^{p} \text { and } r=\inf _{x \in C} r(x) .
$$

Then we have a unique point $z \in C$ (called the asymptotic center of the net $\left\{x_{a}\right\}$ in $C$ ) such that $r(z)=r$ and

$$
r(z) \leqslant r(x)-c_{p}\|x-z\|^{p}
$$

for all $x \in C$, where the constant $c_{p}$ is as in Theorem 7.

Theorem 13 Let $p>1$ be a real number and let $E$ be p-uniformly convex Banach space, $C$ a nonempty bounded closed convex subset of E. Suppose $\mathcal{T}=\left\{T_{s}: s \in J\right\}$ is a left reversible semi-topological uniformly $k$-lipschitzian semigroup on $C$ with $k<\left(1+c_{p}\right)^{\frac{1}{p}}$, where the constant $c_{p}$ is as in Theorem 7. Then

$$
F(\mathcal{T})=\left\{x \in C: T_{s} x=x \text { for all } s \in J\right\} \neq \varnothing
$$

and $F(\mathcal{T})$ is a retract of $C$.

Proof We may assume that $k \geqslant 1$ since if $k<1$, the well known Banach Contraction Principle guarantees a fixed point of $\mathcal{T}$.

Define a sequence $\left\{x_{n}\right\} \subset C$ in the following way: $x_{n+1}$ is the asymptotic center of the net $\left\{T_{s} x_{n}\right\}_{s \in J}$ in $C$. Then, by Lemma 12, we have for $x \in C$ and $n=1,2, \ldots$

$$
c_{p}\left\|x-x_{n+1}\right\|^{p} \leqslant \inf _{s} \sup _{t \geqslant s}\left\|T_{t} x_{n}-x\right\|^{p}-\inf _{s} \sup _{t \geqslant s}\left\|T_{t} x_{n}-x_{n+1}\right\|^{p} .
$$


Noting the inequality

$$
\inf _{s} \sup _{t \geqslant s}\left\|T_{t} y-x\right\|^{p} \leqslant \inf _{s} \sup _{t \geqslant s}\left\|T_{a t} y-x\right\|^{p}
$$

is valid for all $x, y \in C$ and every $a \in J$. Putting $x=T_{a} x_{n+1}$ into (10) we get

$$
\begin{aligned}
c_{p}\left\|T_{a} x_{n+1}-x_{n+1}\right\|^{p} & \leqslant \inf _{s} \sup _{t \geqslant s}\left\|T_{t} x_{n}-T_{a} x_{n+1}\right\|^{p}-\inf _{s} \sup _{t \geqslant s}\left\|T_{t} x_{n}-x_{n+1}\right\|^{p} \\
& \leqslant \inf _{s} \sup _{t \geqslant s}\left\|T_{a t} x_{n}-T_{a} x_{n+1}\right\|^{p}-\inf _{s} \sup _{t \geqslant s}\left\|T_{t} x_{n}-x_{n+1}\right\|^{p} \\
& \leqslant\left(k^{p}-1\right) \inf _{s} \sup _{t \geqslant s}\left\|T_{t} x_{n}-x_{n+1}\right\|^{p} \\
& \leqslant\left(k^{p}-1\right) \inf _{s} \sup _{t \geqslant s}\left\|T_{t} x_{n}-x_{n}\right\|^{p}
\end{aligned}
$$

and hence

$$
\begin{aligned}
\left\|T_{a} x_{n+1}-x_{n+1}\right\|^{p} & \leqslant \frac{k^{p}-1}{c_{p}} \inf _{s} \sup _{t \geqslant s}\left\|T_{t} x_{n}-x_{n}\right\|^{p} \\
& \leqslant M^{n+1} \inf _{s} \sup _{t \geqslant s}\left\|T_{t} x_{0}-x_{0}\right\|^{p},
\end{aligned}
$$

where $M=\frac{k^{p}-1}{c_{p}}<1$ by assumption of the theorem. Inserting $x=T_{a} x_{n-1}$ into (10) and in a similar way to above, we obtain

$$
\left\|T_{a} x_{n}-x_{n+1}\right\|^{p} \leqslant \frac{k^{p}}{c_{p}} \inf _{s} \sup _{t \geqslant s}\left\|T_{t} x_{n}-x_{n}\right\|^{p} .
$$

Combining (11) and (12) it follows that

$$
\begin{aligned}
\left\|x_{n+1}-x_{n}\right\|^{p} & \leqslant\left(\left\|x_{n+1}-T_{a} x_{n}\right\|+\left\|T_{a} x_{n}-x_{n}\right\|\right)^{p} \\
& \leqslant 2^{p-1}\left(\left\|x_{n+1}-T_{a} x_{n}\right\|^{p}+\left\|T_{a} x_{n}-x_{n}\right\|^{p}\right) \\
& \leqslant 2^{p-1} \cdot M^{n} \cdot\left(\frac{k^{p}}{c_{p}}+1\right) \cdot \inf _{s} \sup _{t \geqslant s}\left\|T_{t} x_{0}-x_{0}\right\|^{p},
\end{aligned}
$$

which shows that $\left\{x_{n}\right\}$ is Cauchy. Let $z=\lim _{n \rightarrow+\infty} x_{n}$. Then for each $a \in J$ we have

$$
\begin{aligned}
\left\|z-T_{a} z\right\|^{p} & \leqslant\left(\left\|z-x_{n}\right\|+\left\|x_{n}-T_{a} x_{n}\right\|+\left\|T_{a} x_{n}-T_{a} z\right\|\right)^{p} \\
& \leqslant\left((1+k)\left\|z-x_{n}\right\|+\left\|x_{n}-T_{a} x_{n}\right\|\right)^{p} \\
& \leqslant 2^{p-1}\left[(1+k)^{p}\left\|z-x_{n}\right\|^{p}+\left\|x_{n}-T_{a} x_{n}\right\|^{p}\right] \\
& \leqslant 2^{p-1}\left[(1+k)^{p}\left\|z-x_{n}\right\|^{p}+M^{n} \cdot \inf _{s \geqslant s} \sup _{t \geqslant}\left\|T_{t} x_{0}-x_{0}\right\|^{p}\right] \rightarrow 0
\end{aligned}
$$

as $n \rightarrow+\infty$. Therefore $T_{a} z=z$ for all $a \in J$.

Note that if $x_{0}=x$ is a arbitrary point in $C$, then $x_{m}=A^{m} x$ for $m=1,2, \ldots$ and by (13)

$$
\begin{aligned}
\left\|A^{m+1} x-A^{m} x\right\| & \leqslant 2^{p-1} \cdot M^{m} \cdot\left(\frac{k^{p}}{c_{p}}+1\right) \cdot \inf _{s} \sup _{t \geqslant s}\left\|T_{t} x-x\right\|^{p} \\
& \leqslant 2^{p-1} \cdot M^{m} \cdot\left(\frac{k^{p}}{c_{p}}+1\right) \cdot(\operatorname{diam} C)^{p}
\end{aligned}
$$

for $m=1,2, \ldots$ Thus

$$
\sup _{x \in C}\left\|A^{i} x-A^{m} x\right\| \leqslant 2^{p-1} \cdot \frac{M^{m}}{1-M} \cdot\left(\frac{k^{p}}{c_{p}}+1\right) \cdot(\operatorname{diam} C)^{p} \rightarrow 0
$$


if $i, m \rightarrow+\infty$, which implies that the sequence $\left\{A^{m} x\right\}$ converges uniformly to a function

$$
R x=\lim _{m \rightarrow+\infty} A^{m} x, \quad x \in C .
$$

It follows from Lemma 3 and Remark 4 that $R: C \rightarrow C$ is continuous. Moreover

$$
\begin{aligned}
\left\|R x-T_{a} R x\right\|^{p} & \leqslant\left(\left\|R x-A^{m} x\right\|+\left\|A^{m} x-T_{a} A^{m} x\right\|+\left\|T_{a} A^{m} x-T_{a} R x\right\|\right)^{p} \\
& \leqslant\left((1+k)\left\|R x-A^{m} x\right\|+\left\|A^{m} x-T_{a} A^{m} x\right\|\right)^{p} \\
& \leqslant 2^{p-1}\left[(1+k)^{p} \cdot\left\|R x-A^{m} x\right\|^{p}+\left\|A^{m} x-T_{a} A^{m} x\right\|^{p}\right] \\
& \leqslant 2^{p-1}\left[(1+k)^{p} \cdot\left\|R x-A^{m} x\right\|^{p}+M^{m} \cdot \inf _{s} \sup _{t \geqslant s}\left\|T_{t} x-x\right\|^{p}\right] \rightarrow 0
\end{aligned}
$$

as $m \rightarrow+\infty$, and $R x=T_{a} R x$ for all $a \in J$ and $x \in C$. Thus $R$ is a retraction $C$ onto $F(\mathcal{T})$.

Corollary 14 Let $H$ be a Hilbert space, $C$ a nonempty bounded closed convex subset of $H$ and $\mathcal{T}=\left\{T_{s}: s \in J\right\}$ be a left reversible semi-topological uniformly $k$-lipschitzian semigroup on $C$ with $k<\sqrt{2}$. Then $F(\mathcal{T}) \neq \varnothing$ and $F(\mathcal{T})$ is a retract of $C$.

Corollary 15 Let $C$ be a nonempty bounded closed convex subset of $L^{p}$-space $(1<p<\infty)$ and $\mathcal{T}=\left\{T_{s}: s \in J\right\}$ be a left reversible semi-topological uniformly $k$-lipschitzian semigroup on $C$. Suppose $k<\sqrt{p}$ if $1<p \leqslant 2$, and $k<\left(1+c_{p}\right)^{\frac{1}{p}}$ if $2<p<+\infty$ (here $c_{p}$ is as in Theorem $8(b))$. Then $F(\mathcal{T}) \neq \varnothing$ and $F(\mathcal{T})$ is a retract of $C$.

Open Access This article is distributed under the terms of the Creative Commons Attribution Noncommercial License which permits any noncommercial use, distribution, and reproduction in any medium, provided the original author(s) and source are credited.

\section{References}

1. Ayerbe Toledano, J.M., Domínguez Benavides, T., López Acedo, G.: Measures of noncompactness in metric fixed point theory. Birkhäuser Verlag, Basel (1997)

2. Bruck, R.E., Jr.: Properties of fixed-point sets of nonexpansive mappings in Banach spaces. Trans. Am. Math. Soc. 179, 251-262 (1973)

3. Bynum, W.L.: Normal structure coefficients for Banach spaces. Pacific J. Math. 86, 427-436 (1980)

4. Domínguez Benavides, T., Lorenzo Ramírez, T.P.: Structure of the fixed points set and common fixed points of asymptotically nonexpansive mappings. Proc. Am. Math. Soc. 129, 3549-3557 (2001)

5. Goebel, K., Kirk, W.A.: A fixed point theorem for transformations whose iterates have uniform Lipschitz constant. Studia Math. 47, 135-140 (1973)

6. Goebel, K., Kirk, W.A.: Topics in metric fixed point theory. Cambridge University Press, Cambridge (1990)

7. Górnicki, J.: Remarks on the structure of the fixed-point sets of uniformly lipschitzian mappings in uniformly convex Banach spaces. J. Math. Anal. Appl. 355, 303-310 (2009)

8. Lim, T.C.: On the normal structure coefficient and the bounded sequence coefficient. Proc. Am. Math. Soc. 88, 262-264 (1983)

9. Lim, T.C., Xu, H.K., Xu, Z.B.: Some $L^{p}$ inequalities and their applications to fixed point theory and approximation theory. In: Nevain, P., Pinkus, A. (eds.) Progress in Approximation Theory, pp. 602-624. Academic Press, New York (1991)

10. Prus, S.: Geometrical background of metric fixed point theory. In: Kirk, W.A., Sims, B. (eds.) Handbook of Metric Fixed Point Theory, pp. 93-132. Kluwer Academic Publishers, Dordrecht (2001)

11. Sędłak, E., Wiśnicki, A.: On the structure of fixed-point sets of uniformly lipschitzian mappings. Topol. Methods Nonlinear Anal. 30, 345-350 (2007) 
12. Tan, K.K., Xu, H.K.: Fixed point theorems for lipschitzian semigroups in Banach spaces. Nonlinear Anal. 20, 395-404 (1993)

13. Xu, H.K.: Fixed point theorems for uniformly lipschitzian semigroups in uniformly convex Banach spaces. J. Math. Anal. Appl. 152, 391-398 (1990)

14. Xu, H.K.: Inequalities in Banach spaces with applications. Nonlinear Anal. 16, 1127-1138 (1991) 